\title{
Search for neutrino-induced cascades with the AMANDA detector
}

J. Ahrens, ${ }^{1}$ X. Bai, ${ }^{2}$ G. Barouch, ${ }^{3}$ S. W. Barwick,${ }^{4}$ R. C. Bay,${ }^{5}$ T. Becka, ${ }^{1}$ K.-H. Becker, ${ }^{6}$ D. Bertrand, ${ }^{7}$ F. Binon, ${ }^{7}$ A. Biron, ${ }^{8}$ S. Böser, ${ }^{8}$ J. Booth, ${ }^{4}$ O. Botner, ${ }^{9}$ A. Bouchta, ${ }^{8, *}$ O. Bouhali, ${ }^{7}$ M. M. Boyce, ${ }^{3}$ T. Burgess, ${ }^{10}$ S. Carius, ${ }^{11}$ A. Chen, ${ }^{3}$ D. Chirkin, ${ }^{5}$ J. Conrad, ${ }^{9}$ J. Cooley, ${ }^{3}$ C. G. S. Costa, ${ }^{7}$ D. F. Cowen, ${ }^{12}$ A. Davour, ${ }^{9}$ C. De Clercq, ${ }^{13}$ T. DeYoung, ${ }^{3, \dagger}$ P. Desiati, ${ }^{3}$ J.-P. Dewulf, ${ }^{7}$ P. Doksus, ${ }^{3}$ P. Ekström, ${ }^{10}$ T. Feser, ${ }^{1}$ J.-M. Frère, ${ }^{7}$ T. K. Gaisser, ${ }^{2}$ M. Gaug ${ }^{8}$ H. Geenen, ${ }^{6}$ A. Goldschmidt, ${ }^{14}$ A. Hallgren, ${ }^{9}$ F. Halzen, ${ }^{3}$ K. Hanson, ${ }^{3}$ R. Hardtke, ${ }^{3}$ T. Hauschildt, ${ }^{8}$ M. Hellwig, ${ }^{1}$ G. C. Hill, ${ }^{3}$ P. O. Hulth, ${ }^{10}$ K. Hultqvist, ${ }^{10}$ S. Hundertmark, ${ }^{10}$ J. Jacobsen, ${ }^{14}$ A. Karle, ${ }^{3}$ J. Kim, ${ }^{4}$ B. Koci, ${ }^{3}$ L. Köpke, ${ }^{1}$ M. Kowalski, ${ }^{8}$ J. I. Lamoureux ${ }^{14}$ H. Leich, ${ }^{8}$ M. Leuthold,${ }^{8}$ P. Lindahl, ${ }^{11}$ I. Liubarsky, ${ }^{3}$ D. M. Lowder, ${ }^{5,+}$ J. Madsen, ${ }^{15}$ P. Marciniewski, ${ }^{9}$ H. S. Matis,${ }^{14}$ C. P. McParland,${ }^{14}$ T. Messarius, ${ }^{6}$ T. C. Miller, ${ }^{2,8}$ Y. Minaeva,${ }^{10}$ P. Miočinović, ${ }^{5}$ P. C. Mock, ${ }^{4, \|}$ R. Morse, ${ }^{3}$ T. Neunhöffer, ${ }^{1}$ P. Niessen, ${ }^{13}$ D. R. Nygren,${ }^{14}$ H. Ogelman, ${ }^{3}$ Ph. Olbrechts, ${ }^{13}$ C. Pérez de los Heros, ${ }^{9}$ A. C. Pohl, ${ }^{11}$ R. Porrata, ${ }^{4, \pi}$ P. B. Price,${ }^{5}$ G. T. Przybylski, ${ }^{14}$ K. Rawlins,${ }^{3}$ C. Reed, ${ }^{4, * *}$ E. Resconi, ${ }^{8}$ W. Rhode, ${ }^{6}$ M. Ribordy,${ }^{8}$

S. Richter, ${ }^{3}$ J. Rodríguez Martino, ${ }^{10}$ P. Romenesko, ${ }^{3}$ D. Ross, ${ }^{4}$ H.-G. Sander,${ }^{1}$ K. Schinarakis, ${ }^{6}$ T. Schmidt, ${ }^{8}$ D. Schneider,${ }^{3}$ R. Schwarz, ${ }^{3}$ A. Silvestri, ${ }^{4}$ M. Solarz,${ }^{5}$ G. M. Spiczak,${ }^{15}$ C. Spiering,${ }^{8}$ N. Starinsky, ${ }^{3, \dagger}$ D. Steele, ${ }^{3}$ P. Steffen, ${ }^{8}$

R. G. Stokstad, ${ }^{14}$ K.-H. Sulanke, ${ }^{8}$ I. Taboada, ${ }^{16, *}$ L. Thollander, ${ }^{10}$ S. Tilav, ${ }^{2}$ M. Vander Donckt, ${ }^{7}$ W. Wagner, ${ }^{6}$ C. Walck, ${ }^{10}$ C. Weinheimer, ${ }^{1}$ C. H. Wiebusch, ${ }^{8, *}$ C. Widemann, ${ }^{10}$ R. Wischnewski ${ }^{8}$ H. Wissing, ${ }^{8}$ K. Woschnagg, ${ }^{5}$ W. Wu, ${ }^{4}$ G. Yodh, ${ }^{4}$ and S. Young ${ }^{4}$

(AMANDA Collaboration)

${ }^{1}$ Institute of Physics, University of Mainz, Staudinger Weg 7, D-55099 Mainz, Germany

${ }^{2}$ Bartol Research Institute, University of Delaware, Newark, Delaware 19716

${ }^{3}$ Department of Physics, University of Wisconsin, Madison, Wisconsin 53706

${ }^{4}$ Department of Physics and Astronomy, University of California, Irvine, California 92697

${ }^{5}$ Department of Physics, University of California, Berkeley, California 94720

${ }^{6}$ Fachbereich 8 Physik, BUGH Wuppertal, D-42097 Wuppertal, Germany

${ }^{7}$ Université Libre de Bruxelles, Science Faculty CP230, Boulevard du Triomphe, B-1050 Brussels, Belgium ${ }^{8}$ DESY-Zeuthen, D-15735 Zeuthen, Germany

${ }^{9}$ Division of High Energy Physics, Uppsala University, S-75121 Uppsala, Sweden

${ }^{10}$ Department of Physics, Stockholm University, SCFAB, SE-10691 Stockholm, Sweden

${ }^{11}$ Department of Technology, Kalmar University, S-39182 Kalmar, Sweden

${ }^{12}$ Department of Physics, Pennsylvania State University, University Park, Pennsylvania 16802

${ }^{13}$ Vrije Universiteit Brussel, Dienst ELEM, B-1050 Brussel, Belgium

${ }^{14}$ Lawrence Berkeley National Laboratory, Berkeley, California 94720

${ }^{15}$ Physics Department, University of Wisconsin, River Falls, Wisconsin 54022

${ }^{16}$ Department of Physics and Astronomy, University of Pennsylvania, Philadelphia, Pennsylvania 19104

(Received 27 June 2002; published 10 January 2003)

\begin{abstract}
We report on a search for electromagnetic and/or hadronic showers (cascades) induced by a diffuse flux of neutrinos with energies between $5 \mathrm{TeV}$ and $300 \mathrm{TeV}$ from extraterrestrial sources. Cascades may be produced by matter interactions of all flavors of neutrinos, and contained cascades have better energy resolution and afford better background rejection than throughgoing $\nu_{\mu}$-induced muons. Data taken in 1997 with the AMANDA detector were searched for events with a high-energy cascadelike signature. The observed events are consistent with expected backgrounds from atmospheric neutrinos and catastrophic energy losses from atmospheric muons. Effective volumes for all flavors of neutrinos, which allow the calculation of limits for any
\end{abstract}

\footnotetext{
*Present address: CERN, CH-1211, Genève 23, Switzerland.

${ }^{\dagger}$ Present address: Santa Cruz Institute for Particle Physics, University of California, Santa Cruz, CA 95064.

${ }^{\ddagger}$ Present address: MontaVista Software, 1237 E. Arques Ave., Sunnyvale, CA 94085.

${ }^{\S}$ Present address: Johns Hopkins University, Applied Physics Laboratory, Laurel, MD 20723.

"Present address: Optical Networks Research, JDS Uniphase, 100 Willowbrook Rd., Freehold, NJ 07728-2879.

II Present address: L-174, Lawrence Livermore National Laboratory, 7000 East Ave., Livermore, CA 94550.

**Present address: Dept. of Physics, Massachussetts Institute of Technology, Cambridge, MA 02139.

${ }^{\dagger}$ Present address: SNO Institute, Lively, ON, Canada P3Y 1M3.

\# Present address: Dept. de Física, Universidad Simón Bolívar, Apdo. Postal 89000, Caracas, Venezuela.
} 
neutrino flux model, are presented. The limit on cascades from a diffuse flux of $\nu_{e}+\nu_{\mu}+\nu_{\tau}+\bar{\nu}_{e}+\bar{\nu}_{\mu}+\bar{\nu}_{\tau}$ is $E^{2}(d \Phi / d E)<9.8 \times 10^{-6} \mathrm{GeV} \mathrm{cm}^{-2} \mathrm{~s}^{-1} \mathrm{sr}^{-1}$, assuming a neutrino flavor flux ratio of 1:1:1 at the detector. The limit on cascades from a diffuse flux of $\nu_{e}+\bar{\nu}_{e}$ is $E^{2}(d \Phi / d E)<6.5 \times 10^{-6} \mathrm{GeV} \mathrm{cm}^{-2} \mathrm{~s}^{-1} \mathrm{sr}^{-1}$, independent of the assumed neutrino flavor flux ratio.

DOI: 10.1103/PhysRevD.67.012003

PACS number(s): 14.60.Lm, 95.55.Vj, 95.85.Ry, 96.40.Tv

\section{INTRODUCTION}

Neutrinos interact principally via the weak force, posing a detection challenge for neutrino telescopes but bestowing a valuable advantage on the field of neutrino astronomy: neutrino fluxes from astronomical sources are essentially unattenuated even over cosmological distances. In contrast, highenergy gamma rays are absorbed and/or scattered by intervening matter and photons, and high-energy cosmic-rays are deflected by galactic and intergalactic magnetic fields except at the highest energies $\left(>10^{19} \mathrm{eV}\right)$.

We present a search for the fully reconstructed light patterns created by electromagnetic or hadronic showers (cascades) resulting from a diffuse flux of high-energy extraterrestrial neutrinos. We use data collected in 1997 from the Antarctic Muon and Neutrino Detector Array (AMANDA) for this purpose. Demonstrating $\nu$-induced cascade sensitivity is an important step for neutrino astronomy because the cascade channel probes all neutrino flavors, whereas the muon channel is primarily sensitive to charged current $\nu_{\mu}$ and $\bar{\nu}_{\mu}$ interactions. This is particularly relevant in view of the emerging understanding of neutrino oscillations [1-4], in which the flux of $\nu_{\mu}$ would be reduced by oscillations. (The detection of high-energy atmospheric muon neutrinos by AMANDA has been demonstrated by the full reconstruction of Cherenkov light patterns produced by up-going muons [5-7].) Cascades also boast more accurate energy measurement and better separation from background, although they suffer from worse angular resolution and reduced effective volume relative to muons. Importantly, it is straightforward to calibrate the cascade response of neutrino telescopes such as AMANDA at lower energies through use of, e.g., in situ light sources. Furthermore, cascades become increasingly easier to identify and reconstruct as detector volumes get larger, so the techniques presented here have relevance for future analyses performed at larger detectors.

Electron neutrinos can produce cascades with no detectable track via the charged current (CC) interaction and all neutrino flavors can produce cascades via the neutral current (NC) interaction. Cascade-like events are also produced in $\nu_{\tau}$ $\mathrm{CC}$ interactions when the resulting $\tau$ decays into an electron (roughly $18 \%$ branching ratio) or into mesons (roughly $64 \%$ branching ratio) and the $\tau$ energy is below about $100 \mathrm{TeV}$, at which energy the $\tau$ decay length is less than $5 \mathrm{~m}$, so that the shower produced by the neutrino interaction and the shower produced by the $\tau$ decay cannot be spatially resolved by AMANDA. The contribution of $\nu_{\tau}$ to the cascade channel becomes important when flavor oscillations are taken into account for extraterrestrial [8-10] and for atmospheric [11] $\nu$-induced cascades. For extraterrestrial sources, current knowledge of neutrino oscillations suggests a detected neu- trino flavor flux ratio of $\nu_{e}: \nu_{\mu}: \nu_{\tau}:: 1: 1: 1$ following an expected flux ratio of 1:2:0 at the source.

The total light output of an electromagnetic cascade is approximately $10^{8}$ photons/TeV in ice. Hadronic cascades have a light yield about 20\% lower [12]. An electromagnetic cascade develops in a cylinder of about $10-15 \mathrm{~cm}$ in radius (Molière radius) and several meters in length (about $8.5 \mathrm{~m}$ from the vertex of a $100 \mathrm{TeV}$ cascade, essentially all charged particles are below the critical energy). Hadronic cascades have longer longitudinal developments and larger Molière radii. As a sparsely instrumented detector, AMANDA is insensitive to the topological differences between electromagnetic and hadronic cascades. Since the NC interaction has a lower cross section and results in a deposition of less energy than the CC interaction, and since we assume a steeply falling neutrino energy spectrum, at any given energy a very small fraction of the $\nu_{e}$ events are due to NC interactions. Hence their impact on the cascade energy resolution is small, and the energy spectrum of reconstructed cascades closely follows that of the CC $\nu_{e}$ energy spectrum.

In this paper, we present limits on the diffuse fluxes of $\left(\nu_{e}+\nu_{\mu}+\nu_{\tau}+\bar{\nu}_{e}+\bar{\nu}_{\mu}+\bar{\nu}_{\tau}\right)$ and $\left(\nu_{e}+\bar{\nu}_{e}\right)$, assuming a customary $E^{-2}$ power law spectrum at the source. These limits are based on the observation of no events consistent with a diffuse flux of high-energy extraterrestrial neutrinos. We also present effective volumes for all neutrino flavors to facilitate the calculation of a limit for any flux model. (A search for up-going muons produced by a $\nu_{\mu}$ extraterrestrial diffuse flux is presently being conducted and preliminary results have been reported in [13].)

\section{THE AMANDA-B10 DETECTOR}

The data used in this work were taken with the AMANDA-B10 detector in 1997. AMANDA-B10 $[6,7,14]$ was commissioned in 1997 with a total of 302 optical modules (OMs) arranged on 10 strings, at depths between 1500 $\mathrm{m}$ and $2000 \mathrm{~m}$ below the surface of the ice at the South Pole. The strings are arranged in two concentric circles $35 \mathrm{~m}$ and $60 \mathrm{~m}$ in radius, with one string at the center. The OMs in the inner four (outer six) strings have a $20 \mathrm{~m}(10 \mathrm{~m})$ vertical separation. Each OM contains a $20 \mathrm{~cm}$ photo-multiplier tube (PMT) in a spherical pressure vessel. Coaxial cables in the inner four strings and twisted pair cables in the outer six strings provide high voltage to the PMTs and simultaneously transmit their signals to the electronics housed on the surface. The detector is triggered using a majority condition in which an event is recorded if more than 16 modules have a signal (i.e., were "hit") in a $2 \mu$ s time window. A total of $1.05 \times 10^{9}$ events were recorded during an effective live time of 130.1 days. 
The optical properties of the ice have been studied with in situ light sources and with atmospheric muons. These studies have shown that ice at the South Pole is not perfectly homogeneous, but rather consists of horizontal layers corresponding to global climatological conditions in the past, such as ice ages. These layers lead to a modulation of the absorption and effective scattering lengths as a function of depth [15]. Optical properties are also modified by the presence of drillhole bubbles which are created during the drilling and deployment processes.

\section{METHODS FOR CASCADE RECONSTRUCTION}

Simple reconstruction algorithms are initially applied to the data. These methods are used to reduce the data sample size and to seed more sophisticated reconstruction algorithms, while maintaining high passing rates for simulated signal events. For cascades, the mean position of the hit OMs, or center of gravity, is used as the first guess of the position. In order to efficiently reject muons, they too are reconstructed, beginning with a first guess track fit called the line fit [16]. The line fit is an algorithm that assumes that hits can be projected onto a line, and that the particle which produced the hits travels with a velocity $\vec{v}_{\text {line }}$ and has a starting point $\vec{r}_{0}$. The fit minimizes the quantity $\sum_{\mathrm{i}=1}^{\mathrm{N}_{\text {hits }}}\left(\vec{r}_{i}-\vec{r}_{0}\right.$ $\left.-\vec{v}_{\text {line }} \cdot t_{i}\right)^{2}$ as a function of $\vec{r}_{0}$ and $\vec{v}_{\text {line }}$, where $\mathrm{N}_{\text {hits }}$ is the number of hits in the event. These procedures are described in more detail elsewhere $[17,18]$.

After calculating the first guesses, three maximum likelihood methods are used consecutively to reconstruct precisely the cascade vertex position, time, energy and direction. These methods are described below.

\section{A. Single photoelectron vertex position and time reconstruction}

The cascade vertex position and creation time are reconstructed using a maximum likelihood function that takes into account the Cherenkov emission, absorption and scattering of light. This vertex information is required for rejecting potential backgrounds and for subsequent fits for energy and direction. This procedure is quite similar to the algorithms used for muon fitting [17]. A more comprehensive description of the different cascade reconstruction methods can be found in $[18-20]$.

We use a likelihood function having the form

$$
\mathcal{L}_{\vec{x}, t}^{\text {spe }}=\prod_{i=0}^{N_{\text {hits }}} p\left(t_{\text {res }}^{i}, d_{i}\right),
$$

where $t_{\text {res }}=t_{\text {hit }}-t_{\text {Cher }}$ is the difference between observed hit time and expected time for Cherenkov emission without scattering - the time residual —and $p\left(t_{\text {res }}, d\right)$ is the probability of observing a photon at a time residual $t_{\text {res }}$ at a distance $d$ from the emitter. The label "spe" indicates that $\mathcal{L}_{x, t}^{\text {spe }}$ assumes all hits are due to single photoelectrons.

The probability $p\left(t_{\text {res }}, d\right)$ was generated by parametrizing simulations of light propagation in ice. The product in Eq. (1) is calculated using all hit OMs. The maximization of $\mathcal{L}_{\bar{x}, t}^{\mathrm{spe}}$ provides a significant improvement over the center of gravity in the estimation of the vertex position and time of the cascade. The estimate will be further improved by the multiphotoelectron reconstruction descrived below.

\section{B. Multi-photoelectron vertex position and time reconstruction}

The single photoelectron likelihood can be refined by taking into account that the time measured in each PMT is the time of the first photon to be observed. If a PMT receives $N$ photons, the probability of measuring a time residual, $t_{\text {res }}$, and the associated likelihood function are

$$
\begin{gathered}
p\left(N, t_{\mathrm{res}}, d\right)=N p\left(t_{\text {res }}, d\right)\left(\int_{t_{\text {res }}}^{\infty} d t^{\prime} p\left(t^{\prime}, d\right)\right)^{N-1} \\
\mathcal{L}_{\vec{x}, t}^{\mathrm{mpe}}=\prod_{i=0}^{N_{\text {hits }}} p\left(N, t_{\mathrm{res}}^{i}, d_{i}\right)
\end{gathered}
$$

where the "mpe" label indicates that $\mathcal{L}_{\vec{x}, t}^{\text {mpe }}$ describes multiphotoelectron hits. In AMANDA we use the measured pulse amplitude as an estimator for $N$.

The maximization of $\mathcal{L}_{\vec{x}, t}^{\mathrm{mpe}}$ is used to estimate the most likely vertex position $\vec{x}$ and time $t$ of a cascade. The multiphotoelectron vertex reconstruction uses the maximization of $\mathcal{L}_{x, t}^{\mathrm{spe}}$ to seed initial values of cascade vertex position and time.

\section{Energy and direction reconstruction}

Cascade energy and direction are reconstructed using a likelihood function assembled from the probabilities of an OM being hit or remaining unhit assuming a cascade hypothesis:

$$
\mathcal{L}_{E, \hat{n}}=\prod_{i=0}^{\text {Hit OMs }} P_{\mathrm{Hit}}\left(d_{i}, E, \hat{n}\right) \prod_{i=0}^{\text {Unhit OMs }} P_{\mathrm{Unhit}}\left(d_{i}, E, \hat{n}\right) .
$$

Maximization of $\mathcal{L}_{E, \hat{n}}$ provides the most likely value of energy $E$ and direction $\hat{n}$ of a cascade. Note that in principle this procedure also allows for the reconstruction of position (but not time) of a cascade. Monte Carlo studies have shown, however, that the position resolution obtained by maximizing $\mathcal{L}_{E, \hat{n}}$ is not as good as that obtained with $\mathcal{L}_{\bar{x}, t}^{\mathrm{spe}}$ or $\mathcal{L}_{\vec{x}, t}^{\text {mpe }}$.

\section{DETECTOR RESPONSE TO CASCADES}

In the absence of a tagged source of high-energy neutrinoinduced cascades, to understand the response of the detector we rely on in situ light sources, catastrophic energy losses by down-going cosmic-ray muons, and Monte Carlo simulations. The successful reconstruction of these data demonstrate detector sensitivity to cascade signals.

\section{A. Pulsed laser}

A pulsed laser operating at $532 \mathrm{~nm}$ on the surface is used to send light through optical fibers to diffuser balls embedded in the ice close to almost every OM in the detector. A comparison of Monte Carlo and experimental data for these 

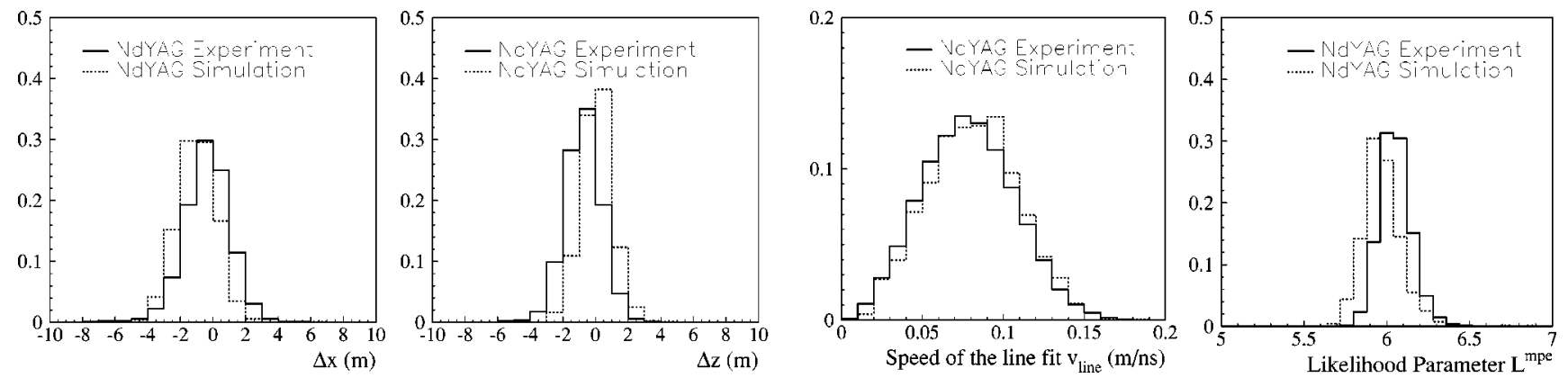

FIG. 1. The figure shows four plots with results of the reconstruction of pulsed laser data (solid) and simulation (dashed) with the diffuser ball next to OM 69 (situated near the center of the detector). The differences between simulated and reconstructed $x$ and $z$ components of the position, the speed of the line fit, $v_{\text {line }}$, and the reduced likelihood parameter, $L_{\vec{x}, t}^{\mathrm{mpe}}$, are shown. The vertical scale for all four plots is arbitrary. The position of the $\mathrm{OM}$ is known with about $1 \mathrm{~m}$ precision [21], so there is no discrepancy in the reconstructed $\Delta z$ plot. The discrepancy in the likelihood parameter plot arises due to the simplified ice model used for the pulsed laser.

in situ light sources deepens our understanding of reconstruction performance and detector signal sensitivity. The photon intensity that can be produced at each diffuser ball is not known a priori, so we force the number of hit channels in experimental and simulated pulsed laser data to match. Thus, the simulations predict that the laser produces pulses in the ice comprising $5 \times 10^{7}-1 \times 10^{9}$ photons (corresponding to a maximum cascade energy of roughly $10 \mathrm{TeV}$ ). The laser pulses are roughly $10 \mathrm{~ns}$ wide, short enough to mimic the time structure of true cascades.

Although highly useful as a cascade calibration source, the pulsed laser system has some minor drawbacks. The diffuser ball light output is expected to be isotropic, so the laser data does not provide information about the angular response of the detector to cascades. The laser produces light at $\lambda$ $=532 \mathrm{~nm}$ and at this wavelength the optical ice properties are different from those at Cherenkov radiation wavelengths. The effective scattering length at $532 \mathrm{~nm}$ is $18-30 \mathrm{~m}$ and depends on depth. The absorption length at $532 \mathrm{~nm}$ is $25 \mathrm{~m}$ and independent of depth [15] (at the shorter wavelengths characteristic of Cherenkov radiation the absorption length is about $100 \mathrm{~m}$ ).

Independent data sets taken with diffuser balls in a variety of locations are reconstructed with the first guesses and with the time-position reconstruction algorithm described above. The position resolution is about $1 \mathrm{~m}$ in the $z$ dimension and about $2 \mathrm{~m}$ the in $x$ and $y$. It is better in $z$ due to closer OM spacing in that dimension.

The pulsed laser simulation uses a simplified optical model of ice properties: the drill-hole bubbles are taken into account, but no depth dependence is used for the scattering length. In spite of this simplification, the vertex resolution of the pulsed laser data agrees well with simulations, showing that the detector can be used to reconstruct the position of contained point-like events. (Contained events are defined as events whose reconstructed vertex lies within a right cylinder of height $400 \mathrm{~m}$ and radius $60 \mathrm{~m}$, centered on the AMANDA-B10 detector and encapsulated by it.) Figure 1

TABLE I. Selection criteria used in the search for high-energy $\nu$-induced cascades with AMANDA-B10. The number of events left after applying each selection criterion to experimental data and background simulations of atmospheric $\mu, \nu_{e}$ and $\nu_{\mu}$ are shown. We simulated 20.3 days of atmospheric $\mu$ data, and 130.1 days of atmospheric $\nu_{e}$ and $\nu_{\mu}$. Signal simulation is also shown for $\nu_{l}+\bar{\nu}_{l}$ and $\nu_{e}+\bar{\nu}_{e}$ assuming $E^{-2}$ spectra, a flux of $1 \times 10^{-4} \mathrm{GeV}^{-1} \mathrm{~cm}^{-2} \mathrm{~s}^{-1} \mathrm{sr}^{-1}$.

\begin{tabular}{lccccccc}
\hline \hline & Selection criteria & Expt. data & Atm $\mu$ & Atm $\nu_{e}$ & Atm $\nu_{\mu}$ & $\nu_{l}+\bar{\nu}_{l}$ & $\nu_{e}+\bar{\nu}_{e}$ \\
\hline \multicolumn{1}{c}{ Trigger } & $1.05 \times 10^{9}$ & $1.51 \times 10^{8}$ & 369.9 & 245.5 & 12446 & 12150 \\
1 & $N_{\text {big TOT }} \geqslant 6$ & & & & & & \\
2 & $v_{\text {line }}<0.12$ or $N_{\text {hits }} \geqslant 75$ & & & & & & \\
3 & $\lambda_{1} / \lambda_{3}>0.35$ & & & & & & \\
4 & $N_{\text {dir }}^{\text {spe }} \geqslant 8$ or $N_{\text {hits }} \geqslant 75$ & & & & & & \\
5 & $L_{x, t}^{\text {spe }}<7.4$ or $N_{\text {hits }} \geqslant 75$ & $5.57 \times 10^{6}$ & $1.12 \times 10^{6}$ & 51.1 & 40.4 & 2727 & 3424 \\
\hline 6 & $L_{\vec{x}, t}^{\mathrm{mpe}}<7.1$ & $1.50 \times 10^{6}$ & $2.03 \times 10^{5}$ & 38.2 & 30.7 & 2128 & 2818 \\
7 & $N_{\text {dir }}^{\mathrm{mpe}} \geqslant 12$ & $1.26 \times 10^{6}$ & $1.14 \times 10^{5}$ & 32.1 & 26.2 & 1909 & 2520 \\
8 & $\theta_{\mu}>80^{\circ}$ & $3.62 \times 10^{5}$ & $2.47 \times 10^{4}$ & 22.5 & 18.5 & 1105 & 1676 \\
9 & Slices in $z_{c}$ & $1.48 \times 10^{5}$ & $1.19 \times 10^{4}$ & 10.6 & 8.5 & 528 & 711 \\
10 & $\cos \left(\theta_{c}\right)<-0.6$ & 675 & 84 & 1.3 & 1.0 & 106 & 156 \\
11 & $E_{c}$ vs $\rho^{\text {mpe }}$ & 0 & 0 & 0.01 & 0.01 & 28.7 & 43 \\
\hline \hline
\end{tabular}




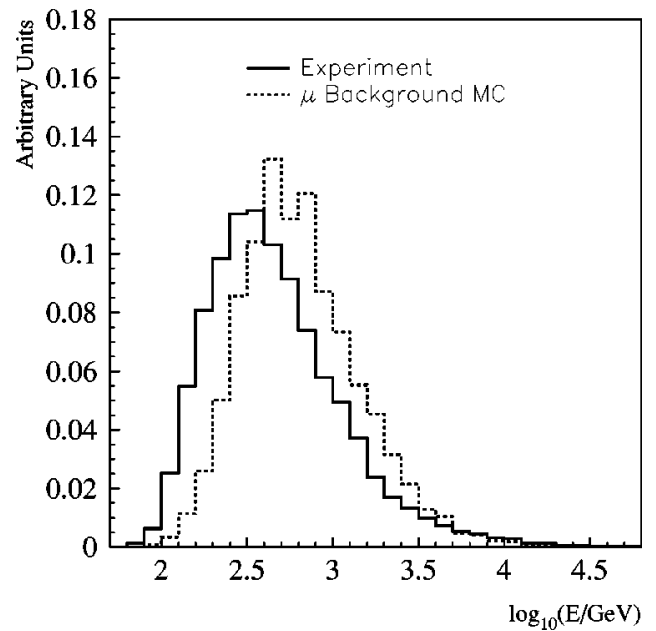

FIG. 2. Energy spectra of reconstructed atmospheric muon energy losses for experimental data and standard simulated muon background after application of selection criteria 1-9 from Table I. Agreement between simulation and experimental data depends on a combination of the simulated ice properties and the OM angular and absolute sensitivity. These effects have been taken into account in the calculation of systematic uncertainties in Sec. VI.

shows the results of the reconstruction of pulsed laser data and simulation.

\section{B. Catastrophic muon energy losses}

The vast majority of the events recorded by AMANDA are down-going muons induced by cosmic-ray air showers. This background has been simulated with the CORSIKA program [22] using the average winter air density profile at the South Pole and the QGSJET hadronic model [23] option. The cosmic-ray composition is taken from [24]. The propagation of muons through ice is simulated with the program MUDEDX $[25,26]$. Optical properties of the ice, including depth dependence and drill-hole bubbles, are also simulated.

From the large sample of atmospheric muons it is possible to extract a subset of cascade-like events in which the majority of the recorded hits come from catastrophic, localized energy loss of the muon (e.g., a bright bremsstrahlung). The extraction of these events is achieved using criteria 1-9 from Table I, i.e., we do not require these events to reconstruct as up-going cascades. (We do, however, still reject obvious down-going muons via criterion number 8.) Based on the number of hits produced by the brightest cascade in simulated events, and on a visual study of these events, we have confirmed that after applying these selection criteria the remaining events are indeed cascade-like.

Figure 2 shows the energy spectra of muon energy losses for experimental data and simulated atmospheric muons. The experimental and simulated data agree reasonably well, but not perfectly. The difference is discussed in Sec. VI.

\section{Monte Carlo prediction for neutrino-induced cascade reconstruction}

To study the performance of the reconstruction algorithms we simulated a flux of $\nu_{e}+\bar{\nu}_{e}$ following an $E^{-2}$ power law spectrum. Neutrinos from astrophysical sources are expected to have a hard spectrum, reflecting the processes in the cosmic accelerators that generate them. Earth absorption and NC scattering are taken into account in the simulation. The "preliminary reference Earth model" is used to calculate the Earth's density profile [27]. We calculate differential cross sections using CTEQ5 following Gandhi et al. [28]. For $\nu_{\tau}$ interactions, the simulation of the $\tau$ decay uses TAUOLA [2931].

Neutrino-induced cascades are reconstructed following the procedure described in Sec. III. Position, zenith angle and energy resolutions for a flux of $\nu_{e}+\bar{\nu}_{e}$ are calculated using the difference distributions shown in Fig. 3. The position resolution is roughly $4 \mathrm{~m}$ in the $z$ dimension and $5.5 \mathrm{~m}$ in $x$ and $y$ for contained cascades. (Note that position resolution obtained with the pulsed laser is better than that predicted for Cherenkov light because optical ice properties are more favorable at the longer wavelength.) The reconstructed position is biased in the direction of the cascade, but since the mean of this shift is only about $2 \mathrm{~m}$ for contained cascades it has a negligible impact on the final result. Zenith angle resolution is $25^{\circ}-30^{\circ}$ depending on the cascade energy.

The energy reconstruction has a resolution of $0.12-0.20$ in $\log _{10} E$ for contained cascades in the range $1 \mathrm{TeV}-100$ $\mathrm{TeV}$, increasing as a function of cascade energy. Energy reconstruction of contained cascades is possible from approximately $50 \mathrm{GeV}$ (the minimum energy cascade which can trigger the detector) to about $100 \mathrm{TeV}$. At energies higher than $100 \mathrm{TeV}$ all, or almost all, of the OMs are hit and thus energy reconstruction by the minimization of Eq. (4) is not
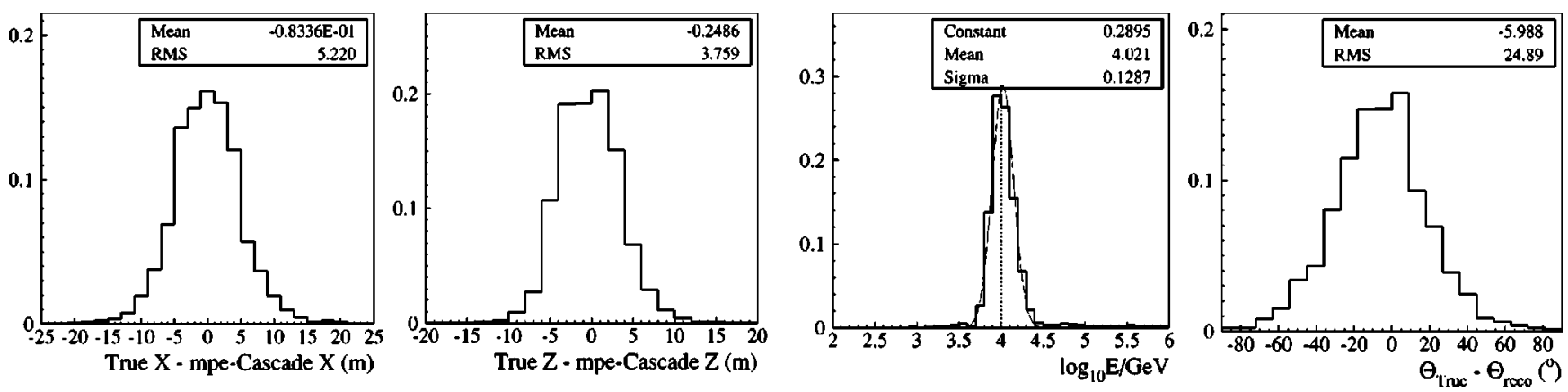

FIG. 3. The four plots show the difference between simulated and reconstructed vertex position, energy and direction of cascades. The monoenergetic cascades have $E=10 \mathrm{TeV}$ and are contained within the detector. Vertical scale for all four plots is arbitrary. 
possible. (Such high-energy events would, however, certainly be identifiable, and probably they can be reconstructed by other techniques.)

\section{ANALYSIS}

\section{A. Filter}

The first step in the analysis is to apply an initial set of selection criteria, here called the "filter," which results in a reduction of the data sample size by more than two orders of magnitude. The filter first removes spurious hits arising from electronic and PMT noise. It then uses the fast reconstruction algorithms described earlier, a simple energy estimator, and topological characteristics of the hit pattern to select potential signal events. The various filter steps were tuned to reject a simulated background of down-going cosmic-ray muons.

After the filter has been applied, hits likely to have come from cross-talk are removed, as explained below. Then each event is reconstructed twice, first with a cascade hypothesis and then with a muon hypothesis. Several selection criteria are then applied to the data based on the results of the reconstruction.

Atmospheric $\nu_{e}$ and $\nu_{\mu}$ neutrinos are simulated according to the flux calculated by Lipari [32]. Contributions to cascades from both atmospheric $\nu_{e}$ (CC and NC interactions) and $\nu_{\mu}$ (NC interactions) are taken into account. In the energy range relevant to this analysis $(E>5 \mathrm{TeV})$, neutrino oscillations are not important in the simulation of atmospheric neutrinos.

\section{B. Removal of electronic cross-talk}

Electronic cross-talk is present in the twisted pair cables used for strings 5-10. Spurious hits arising from cross-talk can degrade the reconstruction quality. Cross-talk is not included in the simulations, so its removal is an important facet of this analysis.

We generate a detector-wide map of the cross-talk using the pulsed laser. For each OM in strings 5-10, pulsed laser data are taken in which only the PMT in the OM near the laser diffuser ball has its high voltage enabled. Any hit in the flashed OM is thus known to be due to light and any hit in any other OM is known to be due to cross-talk. The crosstalk map identifies the pairs of cross-talk-inducing and crosstalk-induced OMs as well as the correlation in time and amplitude of real and cross-talk hits. The map shows that crosstalk occurs for OMs in the same string that are close neighbors or for OMs in the same string that are separated by $150-200 \mathrm{~m}$. The origin of cross-talk is correlated with the relative positioning of individual electrical cables within the string [33].

Cross-talk hits may also be characterized by narrowness (small time over threshold or "TOT") coupled with unexpectedly large amplitude. Cross-talk-induced and lightinduced hits lie in different regions of the amplitude vs TOT space and may therefore be separated from one another. Figure 4 shows the amplitude vs TOT distributions for both types of hits.

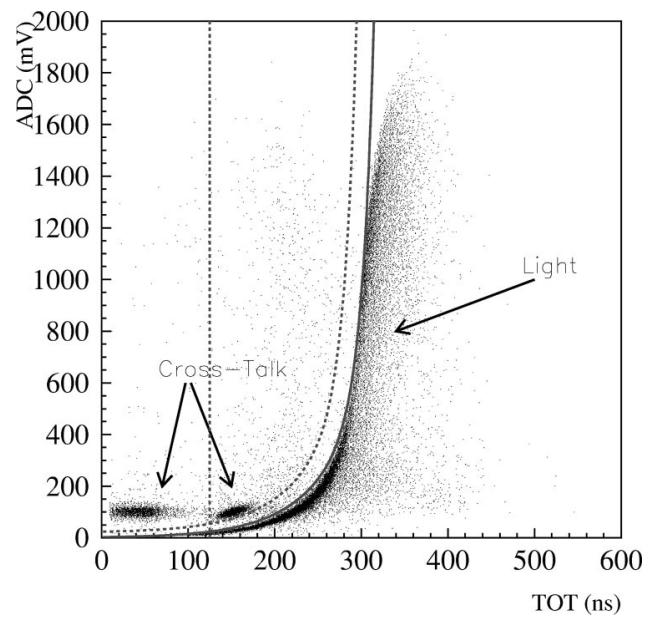

FIG. 4. Amplitude vs time over threshold (TOT) distribution for hits on OM 149 due to light and cross-talk. The data shown to the right of the solid curve are generated using the pulsed laser with only the high voltage for the PMT in OM 149 enabled (all other PMTs had their high voltage disabled). This region of the plot therefore contains hits created by light. The data shown to the left of the solid curve are also generated using the pulsed laser, but with a diffuser ball located $200 \mathrm{~m}$ above OM 149, close to OM 129, in the same string as OM 149. Only OM 129 had its high voltage enabled. This region of the plot therefore contains hits in OM 149 due to cross-talk. Hits are removed from an event if they lie to the left of the dashed curve or if they have a TOT smaller than 125 ns (indicated by the dashed line).

The cross-talk map and the amplitude vs TOT information are both used to remove cross-talk from the experimental data.

\section{Selection criteria}

Table I lists the selection criteria and the passing rates for experimental data and the various samples of Monte Carlo calculations used in this analysis. Selection criteria which have not already been described in Sec. III are described below, followed by a physical justification for each criterion.

The ratio of the smallest to the largest eigenvalues of the tensor of inertia of the position of the hits, $\lambda_{1} / \lambda_{3}$ or sphericity, is used to classify events. ${ }^{1}$ Small values of the sphericity correspond to hits located along a narrow cylinder, as expected for a muon. Values of the sphericity close to unity correspond to a spherical distribution of the hits, as expected for contained cascades.

The dispersive nature of the ice and of the cables that transmit the electrical signals from the OMs to the electronics on the surface render sharp signals in the ice into significantly broader pulses at the surface. For this reason, counting the number of OMs with large TOT, $N_{\text {big TOT }}$, gives a rough estimate of the energy of the event. For this analysis a TOT is considered large if it exceeds the value estimated from Monte Carlo simulations to correspond to one photoelectron by at least a factor of 1.5 . A contained cascade with $300 \mathrm{GeV}$

\footnotetext{
${ }^{1}$ To calculate a tensor of inertia in this context, a unit mass is hypothesized at each hit OM position.
} 


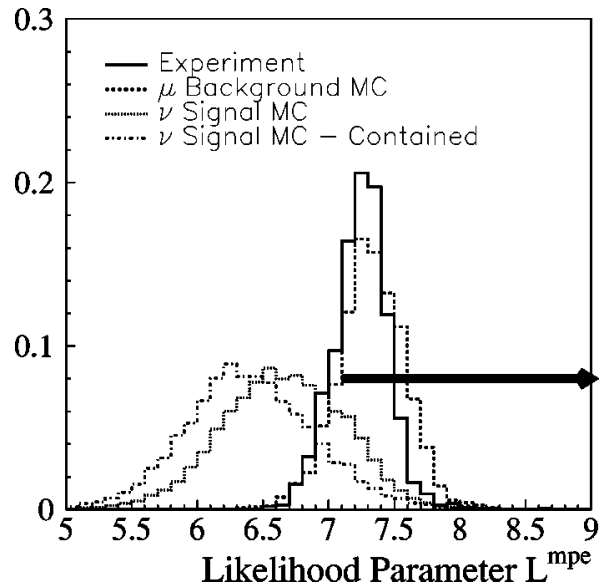

FIG. 5. Normalized distribution of the reduced likelihood parameter $L_{\vec{x}, t}^{\mathrm{mpe}}$ for experimental data (solid line), background atmospheric $\mu$ simulation (dashed line), $\nu_{e}$ simulation assuming an $E^{-2}$ power law spectrum (dotted line) and contained $\nu_{e}$ simulation assuming an $E^{-2}$ power law spectrum (dotted-dashed line). Contained events have their vertices in a cylinder $400 \mathrm{~m}$ in height and $60 \mathrm{~m}$ in radius, roughly matching the detector dimensions. Selection criteria 1-5 from the same table have already been applied to all the samples shown. The arrow indicates the region removed by cut 6 from Table I.

of energy corresponds roughly to $N_{\text {big тОт }}=6$.

The quality of the likelihood reconstruction is determined from the reduced likelihood parameter, defined by $L=$ $-\log \mathcal{L} /\left(N_{\text {hits }}-N_{\text {fit }}\right)$, where $N_{\text {fit }}$ is the number of fitted parameters. Lower values of $L$ correspond to better reconstruction quality.

A hit is considered direct if the time residual is between $-15 \mathrm{~ns}$ and $75 \mathrm{~ns}$. The number of direct hits, $N_{\mathrm{dir}}$, is another measure of the quality of the reconstruction. Both the single and multi-photoelectron position-time reconstruction report the number of direct hits.

Criteria 1-5 of Table I correspond to the filter and these criteria must be satisfied by all events in the analysis. The cut on $N_{\text {big тот }}$ selects high-energy events, and the cut on the sphericity selects events in which the hit topology is cascadelike. The cut on the speed of the line fit, $v_{\text {line }}$, removes easily identified down-going muons. As shown in Fig. 5 and Fig. 6, cuts on the likelihood parameter (criterion 6) and the number of direct hits (criterion 7) are used to eliminate non-cascadelike events and preserve cascade-like events with good reconstruction quality. In addition to the filter criteria, angular cuts (criteria 8 and 10) are used to reduce clear muon-like events and to select up-going cascade-like events, and criterion 11 selects high-energy events within a given distance from the vertical axis of the detector, where $E_{c}$ is the reconstructed cascade energy using Eqs. (3) and (4), and $\rho^{\text {mpe }}$ $=\sqrt{\left(x^{\mathrm{mpe}}\right)^{2}+\left(y^{\mathrm{mpe}}\right)^{2}}$. (Criterion 9 is discussed in detail below.)

The filter was developed based strictly on the predictions of the signal and background Monte Carlo simulations. As more and more cuts were applied after the filter, it was found that the experimental data and simulations disagreed in the shape of the $z$ component of the reconstructed cascade posi-

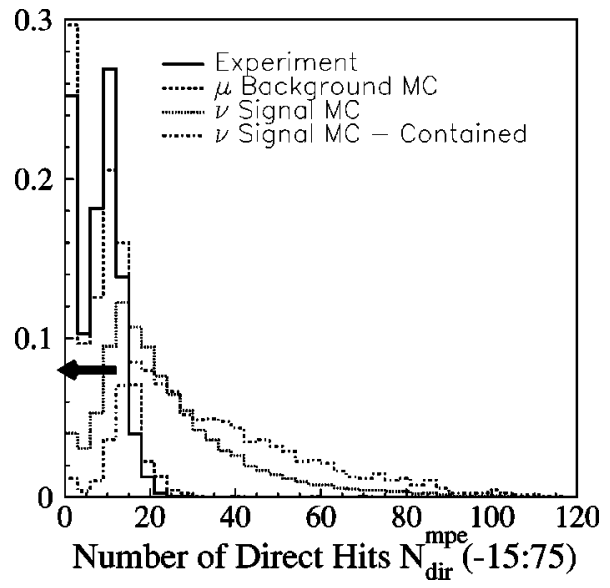

FIG. 6. Normalized distribution of direct hits for experimental data (solid line), background atmospheric $\mu$ simulation (dashed line), $\nu_{e}$ simulation assuming an $E^{-2}$ power law spectrum (dotted line) and contained $\nu_{e}$ simulation assuming an $E^{-2}$ power law spectrum (dotted-dashed line). Contained events have their vertices in a cylinder $400 \mathrm{~m}$ in height and $60 \mathrm{~m}$ in radius, roughly matching the detector dimensions. Selection criteria 1-5 from the same table have already been applied to all the samples shown. The arrow indicates the region removed by cut 7 from Table I.

tion and in the reconstructed cascade direction. Inadequately simulated or unsimulated detector instrumentals, such as optical properties of the ice and cross-talk, contribute to this disagreement. Restricting the regions of the detector used in this analysis reduces the effective volume by more than a factor of two, but restores the agreement between experimental data and simulations. Moreover, the disagreement is also present in the reconstructed cascade direction. Only the regions of the detector that are accepted, show agreement in the reconstructed cascade direction. Events are accepted only if their reconstructed vertices satisfy selection criterion number 9: $-80 \mathrm{~m} \leqslant z_{c} \leqslant-40 \mathrm{~m}$ or $40 \mathrm{~m} \leqslant z_{c} \leqslant 160 \mathrm{~m}$ with respect to the center of the detector (located at $1730 \mathrm{~m}$ below the surface).

\section{SYSTEMATIC UNCERTAINTIES}

There are several uncertainties inherent in estimating the detector sensitivity to high-energy neutrino-induced cascades. First, the detection medium is a natural material (South Pole ice) whose properties are not precisely known. Second, there are no sufficiently powerful accelerator-based sources of neutrinos available for use as calibration beams. Consequently, the understanding of the detector sensitivity is achieved using down-going atmospheric muons, in situ light sources and Monte Carlo simulations.

To estimate the systematic uncertainty due to imprecise knowledge of the optical properties of the ice, simulations have been performed using the least and the most transparent ice that we have measured at AMANDA depths. The cascade sensitivity is modified by $20 \%$ using either extreme model of the optical properties. Uncertainties in the bubble density in the drill-hole ice translate to uncertainties in the OM angular sensitivity. Monte Carlo simulations with increased bubble density in the drill-hole ice degrade the cascade sensitivity 
by $9 \%$. The absolute sensitivity of the OMs is also uncertain at the level of $40 \%$. Monte Carlo simulations with altered absolute OM sensitivity modifies the cascade sensitivity by $5 \%$. This dependence is weak due to the high $N_{\text {hits }}$ requirement imposed by this analysis. (The dependence is much stronger at earlier stages of the analysis, where the average $N_{\text {hits }}$ is much lower. For example, before the filter is applied a variation in absolute OM sensitivity of $40 \%$ results in a modification of the cascade sensitivity by roughly $35 \%$.)

Cross-talk can reduce the sensitivity of the detector to high-energy neutrino-induced cascades. Events for which cross-talk is not fully removed are typically misreconstructed and are therefore unlikely to have sufficient quality to pass our selection criteria. The pulsed laser data is used to estimate the cascade sensitivity loss due to cross-talk for different locations in the detector. These studies indicate that the sensitivity is degraded by $7 \%$ due to cross-talk. (This $7 \%$ degradation is applied directly to the limit and not treated as a systematic uncertainty.) Related to cross-talk is the uncertainty in the limits due to using slices in $z_{c}$. Changing each boundary of the slices by the position resolution in $z$ modifies the cascade sensitivity by $4 \%$.

Uncertainties in the limits due to neutrino-nucleon cross sections, total cascade light output, and cascade longitudinal development have also been estimated using Monte Carlo simulations. For each of these cases the cascade sensitivity is modified by $<5 \%$.

The systematic uncertainties discussed so far are added in quadrature, giving an overall systematic uncertainty on the sensitivity of $25 \%$. We follow the procedure described in $[34,35]$ to determine how to modify the final limit in light of this systematic uncertainty, assuming that the uncertainties are of a Gaussian nature.

The spectrum of cascade-like events produced by downgoing muons is shown in Fig. 2 (see also Sec. IV B). Standard simulations as well as simulations with modified ice properties and $\mathrm{OM}$ angular and absolute sensitivities have been performed. The disagreement between experiment and simulations may be explained by the uncertainties in the knowledge of the optical properties of ice, the OM sensitivity, the cosmic-ray spectrum and the rate of muon energy losses. From Fig. 2 it can be seen that reasonable agreement between experiment and simulations is restored by shifting the energy scale by up to 0.2 in $\log _{10} E$. This uncertainty in the energy scale results in an uncertainty on the sensitivity of less than 25\%. This uncertainty is not independent of the other sources of systematic uncertainty that we have studied. It demonstrates, however, that the overall systematic uncertainty has not been grossly under- or overestimated.

\section{RESULTS}

The analysis is applied to simulated samples of atmospheric $\nu_{e}$ and $\nu_{\mu}$ background, high-energy neutrino signal (all flavors), and atmospheric muons, and to the 1997 experimental data set. In the experimental data zero events are found. The simulation of atmospheric $\nu_{e}$ predicts 0.01 events, and the simulation of atmospheric $\nu_{\mu}$ predicts 0.01 events from $\mathrm{NC}$ interactions (both these numbers have been rounded up from distinct smaller values). Zero events are found in the simulated atmospheric muon sample after all cuts. A limit on the flux of neutrinos assuming an $E^{-2}$ power law spectrum is set using the following formula:

$$
E^{2} \frac{d \Phi}{d E}=\frac{N_{90 \%}}{T N_{A} \rho_{\mathrm{ice}} \sum_{l} f_{l} \int E^{-2} \xi_{l}(E, \theta) \sigma_{\mathrm{tot}}^{l}(E) V_{\mathrm{eff}}^{l}(E, \theta) d \Omega d E}
$$

where $l$ is the neutrino flavor, $E$ the neutrino energy, $\theta$ the neutrino zenith angle, $N_{90 \%}=2.62$ determined using the unified Feldman-Cousins procedure [36] with a correction applied for the estimated $25 \%$ systematic uncertainty $[34,35], T$ the live time (130.1 days), $N_{A}$ Avogadro's number, $\rho_{\text {ice }}$ the density of ice, $\sigma_{\text {tot }}^{l}(E)$ the neutrino cross section [28], $V_{\text {eff }}^{l}(E, \theta)$ the effective volume of the detector (see Table II), $f_{l}$ the fraction of the total neutrino flux comprised by the neutrino flavor $l$, and $\xi_{l}(E, \theta)$ a function that corrects the flux for Earth absorption and $\mathrm{NC}$ scattering. The integration of Eq. (5) has been done for neutrino energies between $5 \mathrm{TeV}$ and $300 \mathrm{TeV}$.

The $90 \%$ C.L. limit on the diffuse flux of $\nu_{e}+\nu_{\mu}+\nu_{\tau}$ $+\bar{\nu}_{e}+\bar{\nu}_{\mu}+\bar{\nu}_{\tau}$ for neutrino energies between $5 \mathrm{TeV}$ and 300 $\mathrm{TeV}$, assuming a neutrino flux ratio of 1:1:1 at the detector, is

$$
E^{2} \frac{d \Phi}{d E}<9.8 \times 10^{-6} \mathrm{GeV} \mathrm{cm}^{-2} \mathrm{~s}^{-1} \mathrm{sr}^{-1} .
$$

The $90 \%$ C.L. limit on the diffuse flux of $\nu_{e}+\bar{\nu}_{e}$ for neutrino energies between $5 \mathrm{TeV}$ and $300 \mathrm{TeV}$ is

$$
E^{2} \frac{d \Phi}{d E}<6.5 \times 10^{-6} \mathrm{GeV} \mathrm{cm}^{-2} \mathrm{~s}^{-1} \mathrm{sr}^{-1} .
$$

The latter limit is independent of the assumed neutrino flux ratio. The limits without incorporating the effects of systematic uncertainties are $9.1 \times 10^{-6} \mathrm{GeV} \mathrm{cm}^{-2} \mathrm{~s}^{-1} \mathrm{sr}^{-1}$ and 6.1 $\times 10^{-6} \mathrm{GeV} \mathrm{cm}^{-2} \mathrm{~s}^{-1 \mathrm{sr}^{-1}}$, respectively. [Note that since the limit in Eq. (6) is on the sum of the fluxes of all neutrino flavors, and the limit in Eq. (7) is on an individual flavor, the former limit should be divided by a factor of three to compare it properly to the latter.]

Our results together with other limits on the flux of diffuse neutrinos are shown in Fig. 8. Since recent results from other low energy neutrino experiments $[1-4]$ indicate that high-energy cosmological neutrinos will have a neutrino fla- 
TABLE II. Effective volume, in units of $10^{-3} \mathrm{~km}^{3}$, for all neutrino flavors as a function of energy and zenith angle after all the selection criteria have been applied. Uncertainties are statistical only.

\begin{tabular}{llcccc}
\hline \hline & & $3.0-10.0 \mathrm{TeV}$ & $10.0-30 \mathrm{TeV}$ & $30-100 \mathrm{TeV}$ & $100-300 \mathrm{TeV}$ \\
\hline & $-1<\cos \theta<-0.6$ & $0.80 \pm 0.05$ & $1.85 \pm 0.10$ & $1.87 \pm 0.15$ & $1.37 \pm 0.20$ \\
$\nu_{e}$ & $-0.6<\cos \theta<-0.2$ & $0.40 \pm 0.03$ & $0.85 \pm 0.07$ & $1.10 \pm 0.10$ & $0.72 \pm 0.10$ \\
& $-0.2<\cos \theta<0.2$ & $0.08 \pm 0.01$ & $0.22 \pm 0.02$ & $0.36 \pm 0.05$ & $0.31 \pm 0.07$ \\
\hline & $-1<\cos \theta<-0.6$ & $0.82 \pm 0.05$ & $1.67 \pm 0.12$ & $1.85 \pm 0.10$ & $1.60 \pm 0.15$ \\
$\bar{\nu}_{e}$ & $-0.6<\cos \theta<-0.2$ & $0.42 \pm 0.03$ & $0.77 \pm 0.07$ & $0.92 \pm 0.07$ & $0.74 \pm 0.10$ \\
& $-0.2<\cos \theta<0.2$ & $0.09 \pm 0.01$ & $0.20 \pm 0.02$ & $0.35 \pm 0.05$ & $0.30 \pm 0.07$ \\
\hline & $-1<\cos \theta<-0.6$ & $0.08 \pm 0.02$ & $0.35 \pm 0.05$ & $0.87 \pm 0.1$ & $1.27 \pm 0.15$ \\
$\nu_{\mu}$ & $-0.6<\cos \theta<-0.2$ & $0.05 \pm 0.01$ & $0.25 \pm 0.03$ & $0.70 \pm 0.10$ & $1.60 \pm 0.10$ \\
& $-0.2<\cos \theta<0.2$ & & & & $0.05 \pm 0.01$ \\
\hline & $-1<\cos \theta<-0.6$ & $0.12 \pm 0.02$ & $0.34 \pm 0.05$ & $0.70 \pm 0.05$ & $1.17 \pm 0.15$ \\
$\bar{\nu}_{\mu}$ & $-0.6<\cos \theta<-0.2$ & $0.05 \pm 0.01$ & $0.25 \pm 0.03$ & $0.70 \pm 0.1$ & $0.14 \pm 0.01$ \\
& $-0.2<\cos \theta<0.2$ & & & & $0.03 \pm 0.01$ \\
\hline & $-1<\cos \theta<-0.6$ & $0.35 \pm 0.05$ & $1.10 \pm 0.10$ & $1.85 \pm 0.15$ & $1.35 \pm 0.20$ \\
$\nu_{\tau}$ & $-0.6<\cos \theta<-0.2$ & $0.15 \pm 0.03$ & $0.50 \pm 0.05$ & $0.85 \pm 0.10$ & $1.05 \pm 0.10$ \\
& $-0.2<\cos \theta<0.2$ & $0.04 \pm 0.01$ & $0.10 \pm 0.02$ & $0.23 \pm 0.05$ & $0.32 \pm 0.07$ \\
\hline & $-1<\cos \theta<-0.6$ & $0.35 \pm 0.05$ & $1.15 \pm 0.10$ & $1.65 \pm 0.10$ & $1.50 \pm 0.15$ \\
$\bar{\nu}_{\tau}$ & $-0.6<\cos \theta<-0.2$ & $0.15 \pm 0.03$ & $0.45 \pm 0.05$ & $0.80 \pm 0.10$ & $1.20 \pm 0.10$ \\
& $-0.2<\cos \theta<0.2$ & $0.06 \pm 0.01$ & $0.12 \pm 0.02$ & $0.22 \pm 0.04$ & $0.31 \pm 0.06$ \\
\hline \hline
\end{tabular}

vor flux ratio of 1:1:1 upon detection, in this figure we scale limits derived under different assumptions accordingly. For example, to do a side-by-side comparison of a limit on the flux of $\nu_{e}+\nu_{\mu}+\nu_{\tau}+\bar{\nu}_{e}+\bar{\nu}_{\mu}+\bar{\nu}_{\tau}$, derived under the assumption of a ratio of $1: 1: 1$, to a limit on just the flux of $\nu_{\mu}+\bar{\nu}_{\mu}$, the latter must be degraded by a factor of three.

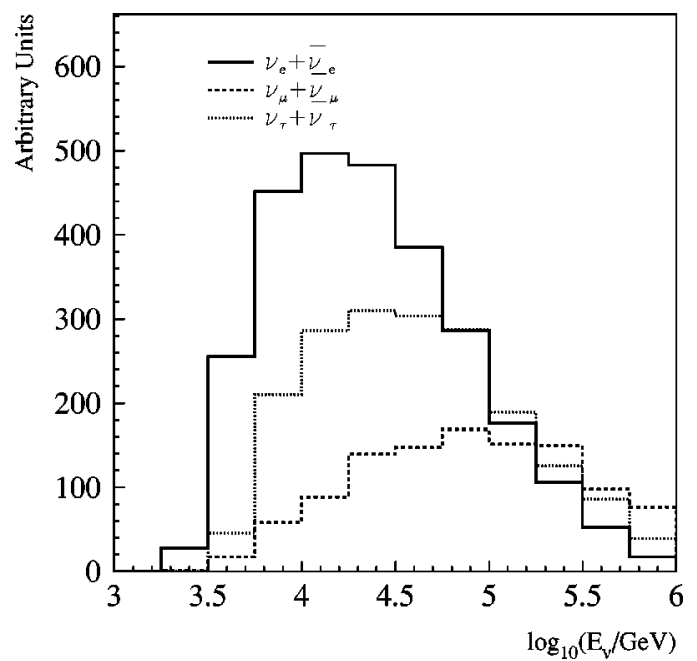

FIG. 7. Distribution of $\nu_{e}, \nu_{\mu}$ and $\nu_{\tau}$ energies after all selection criteria have been applied. The relative normalization between the histograms indicates the relative number of events for each neutrino flavor that passes all the selection criteria. The initial energy distribution follows an $E^{-2}$ spectrum. Neutrino absorption inside Earth, NC scattering and $\tau$ decay have been taken into account as described in Sec. IV C.

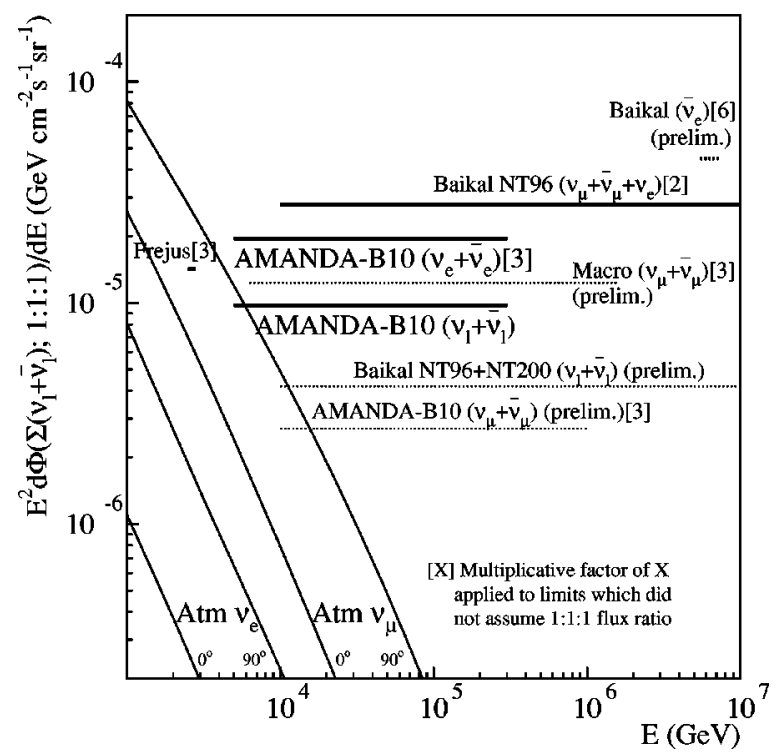

FIG. 8. The limits on the cascade-producing neutrino flux, summed over the three active flavors, presented in this work and in other experiments, with multiplicative factors applied as indicated to permit comparison of limits derived with different assumed neutrino fluxes at the detector: Baikal $\left(\bar{\nu}_{e}\right)$ [38] (at the $W^{ \pm}$resonance); Baikal NT96 $\left(\nu_{\mu}+\bar{\nu}_{\mu}+\nu_{e}\right)$ [39]; Frejus $\left(\nu_{\mu}+\bar{\nu}_{\mu}\right)$ [40]; MACRO $\left(\nu_{\mu}+\bar{\nu}_{\mu}\right) \quad[41] ; \quad$ Baikal NT96+NT200 $\quad\left(\nu_{l}+\bar{\nu}_{l}\right) \quad[38,42]$; AMANDA-B10 $\left(\nu_{\mu}+\bar{\nu}_{\mu}\right)$ [13]. Also shown are the predicted horizontal and vertical $\nu_{e}$ and $\nu_{\mu}$ atmospheric fluxes [32]. 
(N.B.: We assume that $\nu: \bar{\nu}:: 1: 1$.$) Following the Learned and$ Mannheim prescription for presenting limits [37], we show neutrino energy distributions after applying all the selection criteria in Fig. 7.

It should be noted that most searches of diffuse fluxes shown in Fig. 8 are based on the observation of up-going neutrino-induced muons. Only Baikal and AMANDA have presented limits from analyses that search for neutrinoinduced cascades and only the AMANDA analysis uses full cascade event reconstruction.

\section{CONCLUSIONS}

High-energy neutrino-induced cascades have been searched for in the data collected by AMANDA-B10 in 1997. Detailed event reconstruction was performed. Using in situ light sources and atmospheric muon catastrophic energy losses, the sensitivity of the detector to high-energy cascades has been demonstrated.

No evidence for the existence of a diffuse flux of neutrinos producing cascade signatures has been found. Effective volumes as a function of energy and zenith angle for all neutrino flavors have been presented. The effective volumes allow the calculation of limits for any predicted neutrino flux model. The limit on cascades from a diffuse flux of $\nu_{e}+\nu_{\mu}$ $+\nu_{\tau}+\bar{\nu}_{e}+\bar{\nu}_{\mu}+\bar{\nu}_{\tau} \quad$ is $\quad E^{2}(d \Phi / d E)<9.8 \times 10^{-6} \mathrm{GeV} \mathrm{cm}^{-2}$ $\mathrm{s}^{-1} \mathrm{sr}^{-1}$ assuming a neutrino flavor flux ratio of $1: 1: 1$ at the detector. The limit on cascades from a diffuse flux of $\nu_{e}$ $+\bar{\nu}_{e}$ is $E^{2}(d \Phi / d E)<6.5 \times 10^{-6} \mathrm{GeV} \mathrm{cm}^{-2} \mathrm{~s}^{-1} \mathrm{sr}^{-1}$, independent of the assumed neutrino flux ratio. The limits are valid for neutrino fluxes in the energy range of $5 \mathrm{TeV}$ to 300 TeV.

\section{ACKNOWLEDGMENTS}

This research was supported by the following agencies: U.S. National Science Foundation, Office of Polar Programs; U.S. National Science Foundation, Physics Division; University of Wisconsin Alumni Research Foundation; U.S. Department of Energy; Swedish Natural Science Research Council; Swedish Research Council; Swedish Polar Research Secretariat; Knut and Alice Wallenberg Foundation, Sweden; German Ministry for Education and Research; U.S. National Energy Research Scientific Computing Center (supported by the Office of Energy Research of the U.S. Department of Energy); UC-Irvine AENEAS Supercomputer Facility; Deutsche Forschungsgemeinschaft (DFG). D.F.C. acknowledges the support of the NSF CAREER program and C.P. de los H. acknowledges support from the EU 4th framework of Training and Mobility of Researchers.
[1] Q. Ahmad et al., Phys. Rev. Lett. 87, 071301 (2001).

[2] Q. Ahmad et al., Phys. Rev. Lett. 89, 011301 (2002).

[3] Q. Ahmad et al., Phys. Rev. Lett. 89, 011302 (2002).

[4] S. Fukuda et al., Phys. Rev. Lett. 85, 3999 (2000).

[5] E. Andrés et al., Nature (London) 410, 441 (2001).

[6] J. Ahrens et al., Phys. Rev. D 66, 012005 (2002).

[7] J. Ahrens et al., Phys. Rev. D 66, 032006 (2002).

[8] F. Halzen and D. Saltzberg, Phys. Rev. Lett. 81, 4305 (1998).

[9] S. Dutta et al., Phys. Rev. D 64, 113015 (2001).

[10] J. Beacom et al., Phys. Rev. D 66, 021302(R) (2002).

[11] T. Stanev, Phys. Rev. Lett. 83, 5427 (1999).

[12] C. Wiebusch, Ph.D. thesis, RWTH Aachen, Aachen, Germany, 1995.

[13] G. Hill and M. Leuthold, in Proceedings of the 27th International Cosmic Ray Conference, Hamburg, Germany, 2001, p. 1113.

[14] G. Hill, in Proceedings of the 26th International Cosmic Ray Conference, Salt Lake City, 1999, HE.6.3.02.

[15] B. Price et al., Geophys. Res. Lett. 27, 2129 (2000).

[16] V. Stenger, DUMAND Internal Report HDC-1-90, 1990.

[17] C. Wiebusch, DESY-Zeuthen, Germany, 1998, DESY-proc1999-01.

[18] M. Kowalski and I. Taboada, in Proceedings of 2nd Workshop Methodical Aspects of Underwater/Ice Neutrino Telescopes, Hamburg, Germany, 2001.

[19] M. Kowalski, Diploma thesis, Humboldt University, Berlin, Germany, 2000.

[20] I. Taboada, Ph.D. thesis, University of Pennsylvania, Philadelphia, 2002.

[21] E. Andrés et al., Astropart. Phys. 13, 1 (2000).

[22] D. Heck et al., Tech. Rep. FZKA 6019, Forshungszebtrum
Karlsruhe, Karlsruhe, Germany, 1998.

[23] N. Kalmykov et al., Nucl. Phys. B (Proc. Suppl.) 52B, 17 (1997).

[24] B. Wiebel-Sooth et al., astro-ph/9709253.

[25] W. Lohmann et al., CERN Yellow Report CERN-85-03, 1985.

[26] R. Kopp et al. (private comunication).

[27] A. Dziewonski and D.L. Anderson, Phys. Earth Planet. Inter. 25, 297 (1981).

[28] R. Gandhi et al., Phys. Rev. D 58, 093009 (1999).

[29] S. Jadach, J. Kuhn, and Z. Was, Comput. Phys. Commun. 64, 275 (1990).

[30] M. Jezabek, Z. Was, S. Jadach, and J. Kuhn, Comput. Phys. Commun. 70, 69 (1992).

[31] S. Jadach, Z. Was, R. Decker, and J. Kuhn, Comput. Phys. Commun. 76, 361 (1993).

[32] P. Lipari, Astropart. Phys. 1, 193 (1993).

[33] J. Klug, Diploma thesis, Uppsala University, Sweden, 1997.

[34] J. Conrad, O. Botner, A. Hallgren, and C. Perez de los Heros, this issue, Phys. Rev. D 67, 012002 (2003).

[35] J. Conrad, O. Botner, A. Hallgren, and C. Perez de los Heros, Proceedings of Advanced Statistical Techniques in Particle Physics, Durham, 2002, hep-ex/0206034.

[36] G.J. Feldman and R.D. Cousins, Phys. Rev. D 57, 3873 (1998).

[37] J. Learned and K. Mannheim, Annu. Rev. Nucl. Part. Sci. 50, 679 (2000).

[38] V. Balkanov et al., in Proceedings of the 9th International Workshop on Neutrino Telescopes, Venice, Italy, 2001, Vol. II, p. 591, astro-ph/0105269.

[39] V. Balkanov et al., Astropart. Phys. 14, 61 (2000).

[40] W. Rhode et al., Astropart. Phys. 4, 217 (1994).

[41] L. Perrone et al., in Proceedings [13], p. 1073.

[42] Baikal Collaboration, J. Dzhilkibaev (private communication). 